\title{
Reducing PAPR from the Transmitted Signal by the Application of Clipping and Filtering Mechanism
}

\author{
Manmeet Kaur Badwal \\ ECE Department, GNDU RC \\ Jalandhar, India
}

\author{
Harmandar Kaur \\ Assistant Professor \\ ECE Department, GNDU RC \\ Jalandhar, India
}

\begin{abstract}
OFDM is digital multi carrier modulation mechanism used to carry large amount of data from source to destination by following frequency division multiplexing mechanism. Large number of closely related sub carriers is used for carrying data forward. Problem of PAPR starts to originate as more and more data is transferred forward. Peak to average power ratio degrades the performance of the system. In order to overcome the problem hybrid approach of Clipping and Filtering is used. The approach is simulated in MATLAB. The performance analysis indicates better performance as compared to individual approach of SLM and Clipping.
\end{abstract}

\section{Keywords}

OFDM, Modulation, PAPR, SLM, Filtering, Clipping

\section{INTRODUCTION}

OFDM (Orthogonal Frequency division Multiplexing) provides mechanism in order to transfer the data fromsource towards destination. The subcarriers are provided which carry the packets forward. As length of data increases, more and more power dissipation mechanism is utilized in OFDM.This results in PAPR to enhance. Overall performance of the OFDM degrades. The OFDM structure is presented before discussing techniques of tackling PAPR in OFDM[1].

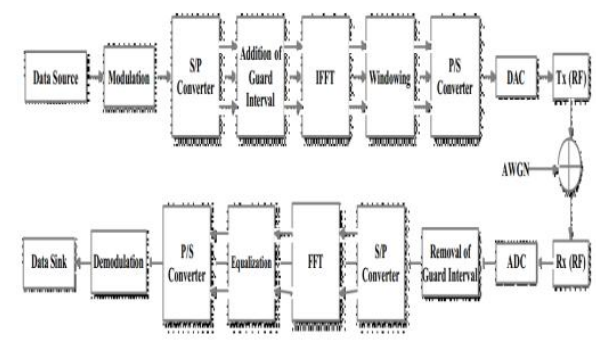

Figure 1. Structure of OFDM for record and play a signal

Components used in OFDM is described as under[2], [3]

\subsection{Serial Data in from data source}

This component act as input component used to receive input in terms of packets. Data is entered sequentially and stored within registers. Register is a combination of flip-flop and is capable of storing values in the form of 0 and 1 . The quantity of register storage depends upon total flip-flops attached to form a register.

\subsection{Frame Formatter}

The source and destination address is entered within packet to form a frame. The frame is transferred by the use of subcarriers in OFDM.

\subsection{OFDM Transmitter}

Frames are received by the OFDM transmitter and modulation is performed to fit the packets in terms of signals within the carriers. Data is forwarded towards destination using OFDM transmitter.

\subsection{Radio channel model}

Transmission channels are divided into sub channels. These channels are radio channels are radio channel model is used for subdivision of channels.

\subsection{D/A converted}

This component is critical as transmitter may or may not be digital in nature but transmission lines are analog. Hence conversion is compulsory in that case. This conversion is performed by the use of D/A Converter.

\subsection{Receiver in terms of Serial Data Output (Sink Nodes)}

Serial data is received by the use of UART (Universal asynchronous resource transmitter).

Serial communication is slow. UART converts serial to parallel and vice versa as required by transmitter and receiver.

These are some of vital components required in order to transfer the data forward. Rest of the paper is organized as under

Section 2 describes literature of existing techniques used in communication in OFDM and PAPR reduction. Section 3 describes the proposed mechanism (Clipping+Filtering), Section 4 describes results and performance analysis, section 5 describes conclusion, section 6 describes references.

\section{LITERATURE SURVEY}

The hybrid approach of clipping and filtering yield better result as compared to existing literature. To prove the same literature survey is conducted.

Selective mapping is the mechanism by which PAPR is reduced from the transferred signals. System complexity and delay increases as PAPR introduced within the system. In orthogonal frequency-division multiplexing (OFDM) systems. SLM requires the transmission of several side information bits for each data block, which results in some data rate loss. These bits must be channel-encoded because they are particularly critical to the error performance of the system[4].

A tone injection strategy for reduction of PAPR in transmitted signal is observed. The approach is capable enough of finding 
optimal value of constellations and hence allowing only energy efficient signals from source toward destination [5].

MSE OFDM is considered in this approach. The primary aim of this approach is to reduce PAPR and accordingly reduce Mean square error from within transmitted signal. The transmission process makes uses large amount of extra bits thus producing low throughput. Some modification in existing approach is thus desired[6]

Wavelet based transformation is proposed to tackle PAPR. The transformation is mechanism of moving signal from source to destination without altering the size and shape of the object. Three main stages are associated with the proposed wavelet system. First of all OFDM transmission system is considered, secondly thresholding mechanism is considered for detecting misinterpreted signal and last filtering is applied to reduce PAPR levels within the transmitted signals[7].

Time slicing approach is used to trim the PAPR from the transmitted signal. Time slice is defined which if expire, rest of the signal is clipped. The process is shifted to next signal is sequence. The time slice is also known as time quantum. The selection of time quantum is critical for the throughput to increase and PAPR to reduce[8].

The study of exiting literature suggests absence of hybrid approach to reduce PAPR from within the signal. Next section describes the proposed scheme for reducing PAPR from within the transmitted signal.

\section{PROPOSED SYSTEM (CLIPPING + FILTERING) FOR PAPR REDUCTION}

The Clipping approach is used to reduce the intensity of the signal to reduce the PAPR from within the transmitted signal. The intensity reduction may cause loss of signal also. Some unwanted noise may be introduced as a result within the transmitted signal. In order to solve the problem PAPR reduction mechanism along with filtering is suggested. The signal representation in Clipping and filtering mechanism is as under[9], [10]
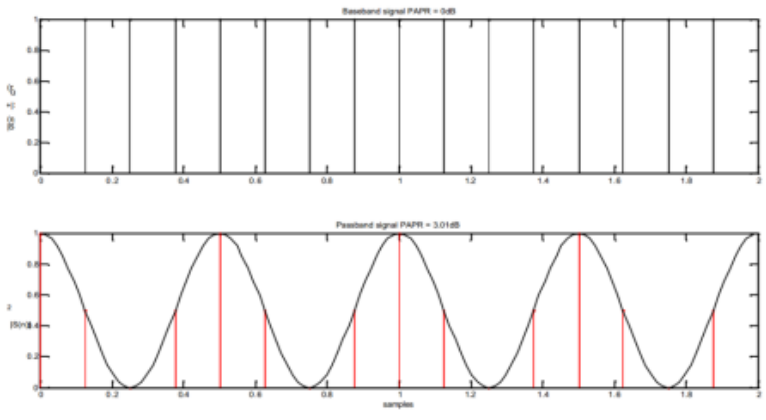

Figure 2. Signal Representation

System model for the Clipping and filtering is as follows

$$
n_{\mathrm{QC}}(t)=\left\{\begin{array}{lc}
\left(|x(t)|-\frac{A_{0}+A_{1}}{2}\right) e^{j \phi(t)}, & A_{0} \leq|x(t)|<A_{1} \\
\left(|x(t)|-\frac{A_{1}+A_{2}}{2}\right) e^{j \phi(t)}, & A_{1} \leq|x(t)|<A_{2} \\
\cdots & \cdots \\
\left(|x(t)|-\frac{A_{m-1}+A_{m}}{2}\right) e^{j \phi(t)}, & A_{m-1} \leq|x(t)|<A_{m} \\
\left(|x(t)|-A_{m}\right) e^{j \phi(t)}, & |x(t)| \geq A_{m}
\end{array}\right.
$$

Equation 1. Quantization and system model followed in clipping and filtering.
Let OFDM signal consist of L subcarriers. Every subcarrier is associated with $\mathrm{T}$ time signal. We have stream of data represented with $\mathrm{D}$. Transmitted signal in that case will be represented as

$$
I(t)=\sum_{b=-\infty}^{\infty} \sum_{n=0}^{L-1} D_{b, n} W_{b, n}(t)
$$

Equation 2.Structure of transmitted signal

$\mathrm{W}_{\mathrm{b}, \mathrm{n}}(\mathrm{t})$ can be described as

$$
W_{b, n}(t)=e^{\frac{2 \pi j n}{T}}\left(t-T_{g}-b T_{c}\right)
$$

Equation 3.Describing $T_{g}$ as guard band and $T_{c}$ as duration of time for signal

The block diagram of clipping and filtering is defined as under

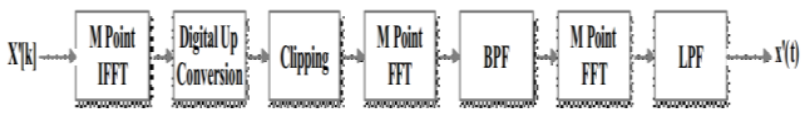

Figure 3. Block diagram of proposed system (Clipping+filtering)

Signals received are passed through inverse Fourier transformation component. The signal once converted is further converted using digital up conversion. The signal falling in the range of PAPR is clipped. The inverse Fourier transformation afterwards is followed by Fourier transformation and filtering. The output signalproduces enhancement in performance and least yielding of PAPR.

\section{RESULTS AND PERFORMANCE ANALYSIS}

This section observes the change in performance and compares the results with existing approach like SLM. The Parameters are given in terms of PAPR reduction parameters

Table 1. Parameter List used in the approach of Clipping and filtering

\begin{tabular}{|c|c|}
\hline Parameters & Value \\
\hline Transmitted Signals & 64 \\
\hline Alphabet Size & 16 \\
\hline L factor & 1.2 \\
\hline QPSK & 64 bits \\
\hline
\end{tabular}

\section{Results are as under}

Table 2. Results in terms of PAPR for various approaches

\begin{tabular}{|c|c|}
\hline Approach & Result(PAPR) \\
\hline Normal OFDM & 22.3743 \\
\hline SLM Modified & 14.9624 \\
\hline Clipped+Filtering OFDM & 11.3356 \\
\hline
\end{tabular}




\section{Results in terms of plots is listed as under}

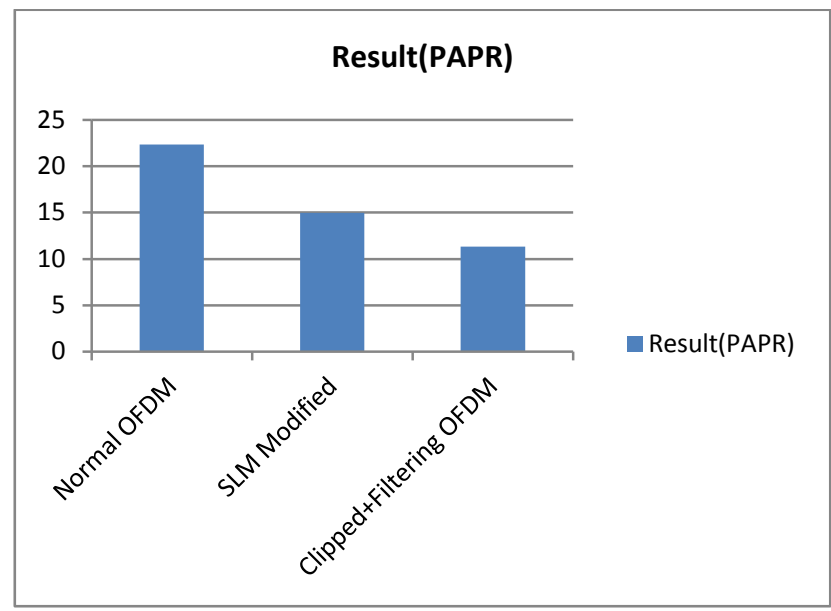

Figure 4. Result in terms of PAPR levels of various approaches.

\section{CONCLUSION AND FUTURE SCOPE}

The proposed approach of Clipping and filtering gives the better result as observed from the performance analysis. The result is improved by almost $20 \%$. The transmission delay introduced due to PAPR is reduced considerably. The PAPR can be further reduced on the application of SLM and filtering approach which is yet to be tested.

\section{REFERENCES}

[1] S. Eom, H. Nam, and Y. Ko, "Low Complexity PAPR Reduction Scheme Without Side Information for OFDM Systems," Signal Process. IEEE Trans., vol. 60, no. 7, pp. 3657-3669, 2012.

[2] A. Jayaprakash and G. R. Reddy, "Covariance Fitting Based Blind Carrier Frequency Offset Estimation Method for OFDM Systems," IEEE Trans. Veh. Technol., vol. PP, no. 99, p. 1, 2016.
[3] A. Zaib, M. Masood, A. Ali, W. Xu, and T. Y. AlNaffouri, "Distributed Channel Estimation and Pilot Contamination Analysis for Massive MIMO-OFDM Systems," IEEE Trans. Commun., vol. 64, no. 11, pp. 4607-4621, 2016.

[4] S. S. Al-Samahi, S. Y. Le Goff, B. S. Sharif, and C. C. Tsimenidis, "A novel OFDM PAPR reduction scheme using selected mapping without explicit side information," IEEE Int. Symp. Pers. Indoor Mob. Radio Commun. PIMRC, vol. 2, pp. 8-12, 2008.

[5] J. Hou, X. Zhao, F. Gong, F. Hui, and J. Ge, "PAPR and PICR Reduction of OFDM Signals with Clipping Noisebased Tone Injection Scheme," IEEE Trans. Veh. Technol., vol. PP, no. 99, p. 1, 2016.

[6] X. Wang, Y. Wu, J. Y. Chouinard, and H. C. Wu, "On the design and performance analysis of multisymbol encapsulated OFDM systems," IEEE Trans. Veh. Technol., vol. 55, no. 3, pp. 990-1002, 2006.

[7] O. Daoud, Q. J. Hamarsheh, and A. A. Damati, "Wavelet Transformation method to allocate the OFDM signals peaks," pp. 159-164.

[8] W. Xianbin, M. J. Rahman, W. Yiyan, T. Bin, Y. Kechu, and J. Y. Chouinard, "A Time Slicing Adaptive OFDM System for Mobile Multimedia Communications," Broadcast. IEEE Trans., vol. 56, no. 2, pp. 226-234, 2010.

[9] H. B. Jeon, J. S. No, and D. J. Shin, "A New PAPR Reduction Scheme Using Efficient Peak Cancellation for OFDM Systems," IEEE Trans. Broadcast., vol. 58, no. 4, pp. 619-628, 2012.

[10] I. Sohn, "A Low Complexity PAPR Reduction Scheme for," vol. 18, no. 2, pp. 225-228, 2014. 